\title{
ELLIPSOIDAL STELLAR SYSTEMS
}

\author{
C. HUNTER
}

The Florida State University, Tallahassee, Fla., U.S.A.

\begin{abstract}
This paper gives a unified account of exact solutions for ellipsoids of uniform density. An approximate solution for a slightly spheroidal system of non-uniform density is given in the final section.
\end{abstract}

\section{Introduction}

Only a very limited number of exact analytical self-consistent solutions for collisionless stellar systems are known that are neither spherically symmetric nor circular disks. Such solutions are the simplest possible models for barred and elliptical galaxies. All the known non-axisymmetric solutions have gravitational potentials that are quadratic functions of the spatial coordinates. Quadratic potentials are given by ellipsoids of uniform density.

The most general ellipsoidal figures of equilibrium of an incompressible fluid of uniform density are the so-called Riemann ellipsoids. Their possible structures and their stability have been studied for the past 200 years, and have given considerable insight into the dynamics of self-gravitating systems. A comprehensive account of this work, with references, is given in Chandrasekhar (1969). Stellar dynamic analogues of these figures are described in the present work.

\section{Triaxial Systems}

Consider an ellipsoid of uniform density $\varrho_{0}$ with outer boundary

$$
\frac{x^{2}}{a_{1}^{2}}+\frac{y^{2}}{a_{2}^{2}}+\frac{z^{2}}{a_{3}^{2}}=1,
$$

that is rotating about the $z$-axis with angular velocity $-\Omega$. A density distribution function that gives a self-consistent solution of the collisionless Boltzmann equation for this figure relative to axes that rotate with the figure, is

$$
f=-\frac{\varrho_{0} a_{1} a_{2} \Lambda^{2}}{\pi^{2} a_{3} k_{\alpha} k_{\beta}\left(2 \pi G \varrho_{0} A_{3}\right)^{1 / 2}} \frac{\mathrm{d}}{\mathrm{d} I}\left[(1-I)^{-1 / 2} H(1-I)\right],
$$

where

$$
I=\frac{x^{2}}{a_{1}^{2}}+\frac{y^{2}}{a_{2}^{2}}+\frac{z^{2}}{a_{3}^{2}}+\Lambda^{2}\left[\frac{a_{2}^{2}\left(\dot{x}+\theta y / a_{2}^{2}\right)^{2}}{k_{\alpha}^{2} k_{\beta}^{2}}+a_{1}^{2}\left(\dot{y}-\theta x / a_{1}^{2}\right)^{2}\right]+\frac{\dot{z}^{2}}{2 \pi G \varrho_{0} a_{3}^{2} A_{3}} .
$$

Here $G$ is the gravitational constant, the dots denote time differentiations, and $k_{\alpha}, k_{\beta}$, $\Lambda^{2}$ and $\theta$ are all constants. Their definitions, as well as details of most of the work described in this section, are given in Hunter (1974). However, the notation for the 
semi-axes and for the coefficients occurring in the gravitational potential such as $A_{3}$ have been changed so as to conform with that of Chandrasekhar (see Chapter 3).

Because the function $H$ in Equation (2) is the Heaviside step function, and differentiation of $H(1-I)$ gives the delta function $-\delta(1-I)$, the distribution function (2) is unphysical since it consists of a positive delta function component and a negative step function component. Hence most orbits must be populated with stars of negative mass if the figure is to be maintained. The total mass of matter at any point in space is positive because of the domination of the delta function component of $f$.

The uniqueness of the solution (2) of the collisionless Boltzmann equation for the given figure has been established rigorously only for the case $\Omega=0$ of no rotation. In Section 3, it is derived again as a solution of the form $f=f(E, J)$ for the spheroidal case, and it seems likely that the solution is unique in the general case. Its unphysical nature must arise from the fact that an excessively large number of stars in high energy orbits are needed to maintain a uniform density right up to the outer boundary. The negative masses are then needed to keep the density uniform in the interior. The present dynamical picture is less general than that of the fluid Riemann ellipsoids that can rotate about an axis other than a principal one but, if more general stellar systems are possible, they will presumably have the same unphysical nature as expression (2).

Negative masses are avoided only in two interesting special cases of Equation (2). The first is that discovered by Freeman (1966a) of $\Omega \rightarrow\left(2 \pi G \varrho_{0} A_{1}\right)^{1 / 2}$. It corresponds to a maximum rate of rotation that the figure can endure because gravitational and centrifugal forces are then in precise balance on the longer $x$-axis $\left(a_{1}>a_{2}\right)$. Any small increase in rotation rate would cause particle orbits to become unstable. Mathematically $k_{\alpha} \rightarrow 0$ in this limit, $\theta \rightarrow 2 \Omega a_{2}^{2}$, and the corresponding limiting form of $f$ must be found. It is in fact

$$
f=\frac{\varrho_{0} \delta(\dot{x}+2 \Omega y) \delta\left(1-J^{\prime}\right)}{\pi \mu \beta a_{2} a_{3}\left[2 \pi G \varrho_{0} A_{3}\right]^{1 / 2}}
$$

where

$$
J^{\prime}=\frac{x^{2}}{a_{1}^{2}}+\frac{y^{2}}{a_{2}^{2}}+\frac{z^{2}}{a_{3}^{2}}+\frac{\left(\dot{y}-2 \Omega a_{2}^{2} x / a_{1}^{2}\right)^{2}}{a_{2}^{2} \mu^{2} \beta^{2}}+\frac{\dot{z}^{2}}{2 \pi G \varrho_{0} a_{3}^{2} A_{3}},
$$

and

$$
\mu \beta=\left(2 \pi G \varrho_{0}\right)^{1 / 2}\left[\left(3-4 a_{2}^{2} / a_{1}^{2}\right) A_{1}+A_{2}\right]^{1 / 2} .
$$

Every populated orbit now touches the outer boundary of the ellipsoid. The particles comprising the ellipsoid have no random motions in the $x$-direction and have a mean motion $\mathbf{u}=\left(-2 \Omega y, 2 \Omega a_{2}^{2} x / a_{1}^{2}, 0\right)$ relative to the ellipsoid.

These figures of Freeman form a two-parameter family of distinct models as they are possible for all values of the ratio of the semi-axes $a_{2} / a_{1}<1$, and for all values of $a_{3} / a_{1}$. In the spheroidal limit $a_{2} \rightarrow a_{1}, \mu \beta \rightarrow 0$, and the random motions in the $y$-direction also disappear. Then all the particles describe circular orbits in the $x-y$ plane with angular velocity $2 \Omega$. In this limit, the distribution function $f$ can be shown to 
become

$$
f=\frac{\varrho_{0} \delta(\dot{x}+2 \Omega y) \delta(\dot{y}-2 \Omega x) H\left(1-J^{\prime \prime}\right)}{\pi a_{3}\left[\left(2 \pi G \varrho_{0} A_{3}\right)\left(1-J^{\prime \prime}\right)\right]^{1 / 2}}
$$

where

$$
J^{\prime \prime}=\frac{x^{2}+y^{2}}{a_{1}^{2}}+\frac{z^{2}}{a_{3}^{2}}+\frac{\dot{z}^{2}}{2 \pi G \varrho_{0} a_{3}^{2} A_{3}} .
$$

The solution given by Bisnovatyi-Kogan and Zel'dovich $(1970, \S 4)$ is precisely this special case of Freeman's solution. Although there are no random motions in the $x$ and $y$-directions, there are random motions which give an anisotropic 'pressure' to support it in the z-direction. Morozov, Polyachenko and Shukman (1974) have recently discussed the stability of these special solutions. It does not seem likely that these solutions are stable, since individual orbits are only marginally stable. Also, mean motions in the figures must be relatively large, so that the figures are liable to the instabilities discussed by Ostriker and Peebles (1973).

The second and dynamically more varied class of solutions without negative masses are the thin disk limits in which $a_{3} \rightarrow 0$ and the ellipsoid is collapsed into a plane perpendicular to the axis of rotation. Then the limit of the distribution function (2) is

$$
f=\frac{3 M \Lambda^{2} H(1-J)}{4 \pi^{2} k_{\alpha} k_{\beta}(1-J)^{1 / 2}}
$$

where $M$ is the mass of the disk, and

$$
J=\frac{x^{2}}{a_{1}^{2}}+\frac{y^{2}}{a_{2}^{2}}+\Lambda^{2}\left[\frac{a_{2}^{2}\left(\dot{x}+\theta y / a_{2}^{2}\right)^{2}}{k_{\alpha}^{2} k_{\beta}^{2}}+a_{1}^{2}\left(\dot{y}-\theta x / a_{1}^{2}\right)^{2}\right] .
$$

These elliptical disks have a surface density distribution

$$
\sigma=\frac{3 M}{2 \pi a_{1} a_{2}}\left(1-\frac{x^{2}}{a_{1}^{2}}-\frac{y^{2}}{a_{2}^{2}}\right)^{1 / 2},
$$

which tends to zero at the outer edge, avoiding the need for negative masses.

These solutions which were also discovered by Freeman (1966b), form a twoparameter family of dynamically distinct figures given by the ranges $0 \leqslant a_{2} / a_{1} \leqslant 1$, for the semi-axes and $0 \leqslant \Omega^{2} / A^{2} \leqslant 1$ for the rotation rate $\left[A^{2}=\lim _{a_{3} \rightarrow 0}\left(2 \pi G \varrho_{0} A A_{1}\right)\right]$. The quantities $a_{2} / a_{1}$ and $\Omega^{2} / A^{2}$ are two basic dimensionless parameters for these systems. The mean flow velocity is now $\mathbf{u}=\left(-\theta y / a_{2}^{2}, \theta x / a_{1}^{2}\right)$, and $\theta$ can range between $\frac{2}{3}$ and -2 . Hence it can be a circulation either in the same sense, or in the opposite sense, to that of the rotation of the figure itself. The quantity $t=-T_{\text {mean }} / W$, where $T_{\text {mean }}$ is the kinetic energy in the form of mean motions and $W$ is the gravitational energy, can vary between the maximum permissible limits 0 and 0.5 . According to Ostriker and Peebles, there is instability to bar-like disturbances if $t$ is greater than 0.14 or thereabouts.

The slow evolution of these disks as they lose mass can also be studied. They tend either to become more circular, or else to become less circular, but non-rotating. 
Interestingly, they also tend to evolve in the direction of decreasing $t$, and hence of increasing stability to bar-like disturbances.

\section{Stellar Maclaurin Spheroids}

The solutions in Section 2 are essentially found by looking for distribution functions that depend on the three isolating integrals available for uniform ellipsoids. These are the $z$-component of the total energy, and two integrals that are connected with the amplitudes of motions in the $x-y$ plane. There is an extra isolating integral when $a_{1}=a_{2}$, and the ellipsoid becomes a uniform spheroid and symmetric about its axis of rotation.

Because of the large number of isolating integrals now present, there must be a large number of possible solutions of the collisionless Boltzmann equation. As with any axisymmetric distribution of mass, we can look for a solution of the form $f\left(E, J^{2}\right)$ where $E=\frac{1}{2} \mathbf{v}^{2}-\psi$ is the total energy, $\mathbf{v}$ the velocity, $\psi$ the gravitational potential, and $J$ the angular momentum about the axis of symmetry. We now work in a fixed frame of reference.

A general method of finding such solutions has been given by Lynden-Bell (1962). It involves first expressing the density $\varrho$ in terms of $\psi$ and $R=\left(x^{2}+y^{2}\right)^{1 / 2}$. Then the Laplace transform of $\partial \varrho / \partial \psi$ with respect to $\psi$ must be taken, and the transform variable labelled $s$. The result, after multiplication by $(s / u)^{1 / 2}$ and setting $R^{2}=s / u$, is the double Laplace transform of $g(B, t)=4 \pi(2 t)^{-1 / 2} f(-B, 2 t)$, when $s$ and $u$ are now regarded as the transform variables for $B$ and $t$ respectively. Hence a double inversion gives $f$. Following these steps, we write

$$
\varrho=\varrho_{0} H\left\{1-\frac{I}{a_{3}^{2} A_{3}}+\frac{\psi}{\pi G \varrho_{0} a_{3}^{2} A_{3}}+\frac{R^{2}}{a_{1}^{2}}\left[\frac{a_{1}^{2} A_{1}}{a_{3}^{2} A_{3}}-1\right]\right\},
$$

since the gravitational potential for the uniform spheroid is

$$
\psi=\pi G \varrho_{0}\left(I-A_{1} R^{2}-A_{3} z^{2}\right) .
$$

The double Laplace transform of $g$ is then obtained as

$$
\varrho_{0}\left(\frac{s}{u}\right)^{1 / 2} \exp \left\{-\pi G \varrho_{0} s\left[I-a_{3}^{2} A_{3}-\frac{s}{a_{1}^{2} u}\left(a_{1}^{2} A_{1}-a_{3}^{2} A_{3}\right)\right]\right\} \text {. }
$$

The inversion of $u^{-1 / 2} \exp (k / u)$ is $(\pi t)^{-1 / 2} \cosh 2(k t)^{1 / 2}$ (Abramowitz and Stegun, 1964, formula 29.3.77). Hence the first inversion of Equation (14) is

$$
\varrho_{0}\left(\frac{s}{\pi t}\right)^{1 / 2} \exp \left\{-\pi G \varrho_{0}\left(I-a_{3}^{2} A_{3}\right) s\right\} \cosh \frac{2 s}{a_{1}}\left[\pi G \varrho_{0} t\left(a_{1}^{2} A_{1}-a_{3}^{2} A_{3}\right)\right]^{1 / 2} \text {. }
$$

As always, negative exponential terms represent a shift of arguments, and the cosh term can be written as the sum of two exponentials. The inversion of $s^{1 / 2}$ leads to a general- 
ized function, since the inversion of $s^{-1 / 2}$ is $(\pi B)^{-1 / 2}$, and the extra $s$ factor corresponds to a differentiation. The result therefore is that

where

$$
\begin{aligned}
g(B, t)= & \frac{\varrho_{0}}{2 \pi t^{1 / 2}} \frac{\mathrm{d}}{\mathrm{d} B}\left\{\left[B-\pi G \varrho_{0}\left(I-a_{3}^{2} A_{3}\right)+\right.\right. \\
& \left.+\omega(2 t)^{1 / 2}\right]^{-1 / 2} H\left[B-\pi G \varrho_{0}\left(I-a_{3}^{2} A_{3}\right)+\omega(2 t)^{1 / 2}\right]+ \\
& +\left[B-\pi G \varrho_{0}\left(I-a_{3}^{2} A_{3}\right)-\omega(2 t)^{1 / 2}\right]^{-1 / 2} H\left[B-\pi G \varrho_{0}\left(I-a_{3}^{2} A_{3}\right)-\right. \\
& \left.\left.-\omega(2 t)^{1 / 2}\right]\right\}
\end{aligned}
$$

$$
\omega=\left[2 \pi G \varrho_{0}\left(A_{1}-a_{3}^{2} A_{3} / a_{1}^{2}\right)\right]^{1 / 2} .
$$

The distribution function is therefore

$$
\begin{aligned}
f\left(E, J^{2}\right)= & |J| g\left(B=-E, t=\frac{1}{2} J^{2}\right) / 4 \pi \\
= & \frac{-\varrho_{0}}{4 \pi^{2} \sqrt{2}} \frac{\mathrm{d}}{\mathrm{d} E^{\prime}}\left[\left(-E^{\prime}+\omega J\right)^{-1 / 2} H\left(-E^{\prime}+\omega J\right)+\right. \\
& \left.+\left(-E^{\prime}-\omega J\right)^{-1 / 2} H\left(-E^{\prime}-\omega J\right)\right],
\end{aligned}
$$

where

$$
E^{\prime}=E+\pi G \varrho_{0}\left(I-a_{3}^{2} A_{3}\right)=\frac{1}{2} \mathbf{v}^{2}+\pi G \varrho_{0}\left[A_{1} R^{2}+A_{3}\left(z^{2}-a_{3}^{2}\right)\right]
$$

This distribution is made up of two equal and oppositely rotating components, and either type of component on its own gives a possible solution. Thus,

$$
f=\frac{-\varrho_{0}}{2 \pi^{2} \sqrt{ } 2} \frac{\mathrm{d}}{\mathrm{d} E^{\prime}}\left[\left(-E^{\prime}+\omega J\right)^{-1 / 2} H\left(-E^{\prime}+\omega J\right)\right],
$$

gives rise to a uniform density spheroid. It is a function only of

$$
-E^{\prime}+\omega J=-\frac{1}{2} v_{R}^{2}-\frac{1}{2}\left(v_{\theta}-\omega R\right)^{2}-\frac{1}{2} v_{z}^{2}+\pi G \varrho_{0} a_{3}^{2} A_{3}\left(1-\frac{R^{2}}{a_{1}^{2}}-\frac{z^{2}}{a_{3}^{2}}\right),
$$

where $\left(v_{R}, v_{\theta}, v_{z}\right)$ denote the components of velocity in cylindrical polar coordinates $(R, \theta, z)$. Hence the distribution function (20) represents a mean flow with angular velocity $\omega$, which is precisely that for a fluid Maclaurin spheroid. The distribution function (20) therefore describes the stellar Maclaurin spheroid. The random velocities are distributed isotropically about the mean flow and provide the necessary pressure support. Unfortunately however, the solution is again unphysical because of widespread negative values of $f$, and is in fact just a particular $a_{1}=a_{2}$ case of solution (2).

\section{A Non-Uniform Stellar Spheroid}

Something other than a uniform density figure must clearly be considered for realistic models of galaxies. Lynden-Bell gave one exact axisymmetric solution as an illustration of his method and indeed, apart from the solution discussed in Sections 2 and 3, it appears to be the only one known. Lynden-Bell's solution is of infinite extent, and is 
a rotationally modified form of the $n=5$ polytrope. A wider variety of known exact solutions would be very desirable, but, unfortunately, they are not easily obtained. The basic difficulty is that a considerable degree of analytical simplicity is necessary if all the integrations involved in Lynden-Bell's method are to be performed, and such simplicity is not often attained. For instance, the spheroid with density,

$$
\varrho=\varrho_{0}\left[1-\frac{R^{2}}{a_{1}^{2}}-\frac{z^{2}}{a_{3}^{2}}\right] H\left[1-\frac{R^{2}}{a_{1}^{2}}-\frac{z^{2}}{a_{3}^{2}}\right],
$$

where $\varrho_{0}$ is now the central density is the most simple spheroid other than the uniform one. Its gravitational potential is still relatively simple and is (Chandrasekhar, 1969, p. 53),

$$
\psi=\frac{1}{2} \pi G \varrho_{0}\left\{I-2 A_{1} R^{2}-2 A_{3} z^{2}+A_{11} R^{4}+2 A_{13} R^{2} z^{2}+A_{33} z^{4}\right\} .
$$

Elimination of $z^{2}$ between Equations (22) and (23) and differentiation of $\varrho$ gives

$$
\frac{\partial \varrho}{\partial \psi}=\left(\pi G a_{3}^{2}\right)^{-1}(\beta \psi-\alpha)^{-1 / 2} H\left[(\beta \psi-\alpha)^{1 / 2}-\gamma\right],
$$

where

$$
\begin{aligned}
& \alpha=I A_{33}-A_{3}^{2}-2 R^{2}\left(A_{1} A_{33}-A_{3} A_{13}\right)+R^{4}\left(A_{11} A_{33}-A_{13}^{2}\right), \\
& \beta=2 A_{33} / \pi G_{0}, \quad \gamma=A_{3}-a_{3}^{2} A_{33}-R^{2}\left(A_{13}-a_{3}^{2} A_{33} / a_{1}^{2}\right) .
\end{aligned}
$$

raking the Laplace transform of expression (24) with respect to $\psi$, and multiplication by $(s / u)^{1 / 2}$ gives as the function to be inverted

$$
\frac{1}{G a_{3}^{2}(\pi \beta u)^{1 / 2}} \exp \left(\frac{-\alpha s}{\beta}\right) \operatorname{erfc} \gamma\left(\frac{s}{\beta}\right)^{1 / 2} .
$$

Since it is necessary to set $R^{2}=s / u$ in the expressions for $\alpha$ and $\gamma$, we obtain for expression (26) a formula that is more complicated than any entry in any of the standard tables. However, expression (26) can be inverted in the spherical case $a_{1}=a_{3}$, and we can progress if we limit our interest to the case of small eccentricity $e=\left(1-a_{3}^{2} / a_{1}^{2}\right)^{1 / 2}$. The following expansions are then valid

$$
\begin{aligned}
\frac{\alpha}{\beta} & =\frac{4 \pi G \varrho_{0} a_{1}^{2}}{9}\left(1-\frac{e^{2}}{7}-\frac{6 e^{2} s}{35 a_{1}^{2} u}\right)+O\left(e^{4}\right), \quad A_{33}=\frac{2}{5 a_{1}^{2}}\left(1+\frac{9 e^{2}}{7}\right)+O\left(e^{4}\right), \\
\frac{\gamma}{\beta^{1 / 2}} & =\left(\frac{4 \pi G \varrho_{0} a_{1}^{2}}{45}\right)^{1 / 2}\left(1-\frac{e^{2}}{14}-\frac{3 e^{2} s}{7 a_{1}^{2} u}\right)+O\left(e^{4}\right) .
\end{aligned}
$$

Upon substitution into expression (26), and expanding for the complementary error function, we obtain

$$
\frac{1}{G a_{3}^{2}(\pi \beta u)^{1 / 2}} \exp \left[-\frac{4 \pi G \varrho_{0} a_{1}^{2} s}{9}\left(1-\frac{e^{2}}{7}\right)+\frac{8 \pi G \varrho_{0} e^{2} s^{2}}{105 u}\right] \times
$$




$$
\begin{aligned}
& \times\left\{\operatorname{erfc}\left[\frac{4 \pi G \varrho_{0} a_{1}^{2} s}{45}\left(1-\frac{e^{2}}{7}\right)\right]^{1 / 2}+\frac{4 e^{2} s^{3 / 2}}{7 a_{1} u}\left(\frac{G \varrho_{0}}{5}\right)^{1 / 2} \times\right. \\
& \left.\times \exp \left[-\frac{4 \pi G \varrho_{0} a_{1}^{2} s}{45}\left(1-\frac{e^{2}}{7}\right)\right]\right\}+O\left(e^{4}\right)
\end{aligned}
$$

The inversion with respect to $u$ can now be carried out. We need the formula used earlier and also Abramowitz and Stegun (formula 29.3.79) that $u^{-3 / 2} \exp (k / u)$ inverts to $(\pi k)^{-1 / 2} \sinh 2(k t)^{1 / 2}$. This leaves us with functions of $s$ that are the products of exponentials with either $s^{1 / 2}$ or $\operatorname{erfc}(k s)^{1 / 2}$. Hence the only new formula needed is Abramowitz and Stegun (formula 29.3.114) shifted that the inverse of $\operatorname{erfc}(k s)^{1 / 2}$ is $k^{1 / 2} H(B-k) /\left[\pi B(B-k)^{1 / 2}\right]$. We then obtain

$$
\begin{aligned}
g(B, t)= & \frac{1}{2 \pi^{2} G a_{3}^{2}}\left(\frac{\lambda}{5 \beta t}\right)^{1 / 2}\left\{\frac{H(B-6 \lambda / 5+\mu \sqrt{2} t)}{(B-\lambda+\mu \sqrt{2 t})(B-6 \lambda / 5+\mu \sqrt{2 t})^{1 / 2}}+\right. \\
& +\frac{H(B-6 \lambda / 5-\mu \sqrt{2 t})}{(B-\lambda-\mu \sqrt{2 t})(B-6 \lambda / 5-\mu \sqrt{2 t})^{1 / 2}}+ \\
& +\frac{e}{a_{1}}\left(\frac{30 t}{7 \lambda}\right)^{1 / 2} \frac{\mathrm{d}}{\mathrm{d} B} \times \\
& \left.\times\left[\frac{H(B-6 \lambda / 5+\mu \sqrt{2 t})}{(B-6 \lambda / 5+\mu \sqrt{2 t})^{1 / 2}}-\frac{H(B-6 \lambda / 5-\mu \sqrt{2 t})}{(B-6 \lambda / 5-\mu \sqrt{2 t})^{1 / 2}}\right]\right\}+O\left(e^{2}\right),
\end{aligned}
$$

where the constants $\lambda$ and $\mu$ are defined by

$$
\lambda=\frac{4 \pi G \varrho_{0} a_{1}^{2}}{9}\left(1-\frac{e^{2}}{7}\right), \quad \mu=4 e\left(\frac{\pi G \varrho_{0}}{105}\right)^{1 / 2} .
$$

The four components of (30) clearly form two pairs. Moreover, each pair combines naturally since, to the first order in any small quantity $\varepsilon$, the Taylor expansion

$$
\begin{aligned}
\frac{\left(1-\frac{1}{2} \varepsilon\right) H[B-c+(\varepsilon-1) \lambda / 5]}{(B-c)[B-c+(\varepsilon-1) \lambda / 5]^{1 / 2}}= & \frac{H(B-c-\lambda / 5)}{(B-c)(B-c-\lambda / 5)^{1 / 2}}+ \\
& +\varepsilon \frac{\mathrm{d}}{\mathrm{d} B}\left[\frac{H(B-c-\lambda / 5)}{(B-c-\lambda / 5)^{1 / 2}}\right]+O\left(\varepsilon^{2}\right),
\end{aligned}
$$

holds for any quantity $c$.

The solution, like that of the previous section, is the sum of two oppositely rotating components, both of which could individually give the full solution if doubled. Taking the first component, we have as a possible solution

$$
f=\frac{\varrho_{0}[1-5 \mu J / 4 \lambda] H\left[-E-6 \lambda / 5+\frac{3}{2} \mu J\right]}{6 \pi^{2} \sqrt{2}(\mu J-\lambda-E)\left[-E-6 \lambda / 5+\frac{3}{2} \mu J\right]^{1 / 2}}+O\left(e^{2}\right) .
$$

Note that expression (33) is correct only to $O(e)$. Although the transformed expression (26) was expanded correctly to $O\left(e^{4}\right)$, the inversion process alters the ordering of the 
expansion in powers of $e$. For instance, the exponential in $e^{2} s / u$ gives rise to effects that are $O(e)$, and an $O\left(e^{2}\right)$ error is introduced with the use of formula (32).

The distribution function (33) has an inverse square root singularity when

$$
\begin{aligned}
& 0=-E-6 \lambda / 5+\frac{3}{2} \mu J \\
& =\frac{\pi G \varrho_{0} a_{1}^{2}}{15}\left(7-\frac{3 r^{2}}{a_{1}^{2}}\right)\left(1-\frac{r^{2}}{a_{1}^{2}}\right)-\frac{1}{2}\left[v_{R}^{2}+\left(v_{\theta}-\frac{3}{2} \mu R\right)^{2}+v_{z}^{2}\right]+O\left(e^{2}\right),
\end{aligned}
$$

where $r=\left(x^{2}+y^{2}+z^{2}\right)^{1 / 2}$. The inverse square root is needed to maintain the correct density in the outer regions, and only orbits with $-E-6 \lambda / 5+\frac{3}{2} \mu J=0$ ever reach the outer edge.

Integration of $f$ over all the allowable velocities confirms that the density at any point in space is $\varrho_{0}\left(1-r^{2} / a^{2}\right)+O\left(e^{2}\right)$, while the mean motion of particles consists of a rotation about the axis of symmetry with angular velocity

$$
\frac{1}{R} \frac{\iiint v_{\theta} f \mathrm{~d} \mathbf{v}}{\iiint f \mathrm{~d} \mathbf{v}}=\frac{3 \mu}{8}\left(5-\frac{r^{2}}{a_{1}}\right)=\frac{3 e}{2}\left(\frac{\pi G \varrho_{0}}{105}\right)^{1 / 2}\left(5-\frac{r^{2}}{a_{1}^{2}}\right) .
$$

This angular velocity, which, as in the case of a fluid spheroid is proportional to the eccentricity, is not uniform, and is somewhat larger at the center than at the edge of the spheroid.

\section{Acknowledgement}

This work has been supported in part by the National Science Foundation under grant GP-34279X.

\section{References}

Abramowitz, M. and Stegun, I. A.: 1964, Handbook of Mathematical Functions, National Bureau of Standards, Washington, D.C., Ch. 29.

Bisnovatyi-Kogan, G. S. and Zel'dovich, Ya. B.: 1970, Astrofizika 6, 387.

Chandrasekhar, S.: 1969, Ellipsoidal Figures of Equilibrium, Yale University Press, New Haven, Conn.

Freeman, K. C. : 1966a, Monthly Notices Roy. Astron. Soc. 134, 1.

Freeman, K. C.: 1966b, Monthly Notices Roy. Astron. Soc. 134, 15.

Hunter, C.: 1974, Monthly Notices Roy. Astron. Soc. 166, 633.

Lynden-Bell, D.: 1962, Monthly Notices Roy. Astron. Soc. 123, 447.

Morozov, A. G., Polyachenko, V. L., and Shukhman, I. G.: 1974, Astron. Zh. 51, 75.

Ostriker, J. P. and Peebles, P. J. E. : 1973, Astrophys. J. 186, 467.

\section{DISCUSSION}

King: There is another interesting application of Maclaurin spheroids that is of great practical interest. If you write down stellar-dynamical equations for the center of a stellar system and expand everything around the origin, then consideration of the leading terms gives a relation between rotation and flattening that is exactly the same as the relation for Maclaurin spheroids.

Fackerell: One suspects that the distribution function in the last example will come out in terms of hypergeometric functions of several variables, probably Lauricella functions.

Hunter: The complications in inverting the Laplace transforms in the Lynden-Bell method are not due to the presence of hypergeometric functions. The functions involved are exponential and complementary error functions, but their arguments are more complicated than those given in current tables.

Lynden-Bell: One trouble with my method is the awkwardness of expressing the density as a function 
of axial radius $R$ and potential $\Phi$. One can avoid some of that trouble if one uses the crazy device of starting not with $\Phi(R, z)$ but with $z(R, \Phi)$. One may differentiate $z(R, \Phi)$ directly to find $\varrho(R, \Phi)$.

I am glad to see that you have managed to get more success in using the method which I found led to complicated functions in many cases.

Hunter: There are two stages at which your method may become difficult. One is that of the algebraic manipulations about which you asked. The other is that of taking the Laplace transforms and then inverting them. I think that this second stage is likely to be the harder one for more general problems. 